\title{
Isolated Neuropathy of the Motor Branch of the Ulnar Nerve Sparing the Hypothenar Muscle and UInar Intrinsic Groups due to Compressive Lesion: A Case Report
}

\author{
Lawrence M Asprec, $M D^{1^{*}}$, Mitchell K Freedman ${ }^{2}$, Pedro K Beredjiklian ${ }^{3}$ and George W Young ${ }^{4}$ \\ ${ }^{1}$ Department of Rehabilitation Medicine, Thomas Jefferson University Hospital, Sidney Kimmel Medical College Philadelphia, \\ USA \\ ${ }^{2}$ Medical Director of Physical Medicine and Rehabilitation, Rothman Orthopaedic Institute, USA \\ ${ }^{3}$ Chief of Hand Surgery, Professor of Orthopaedic Surgery, Rothman Orthopaedic Institute, USA \\ ${ }^{4}$ Department of Physical Medicine and Rehabilitation, Rothman Orthopaedic Institute, USA
}

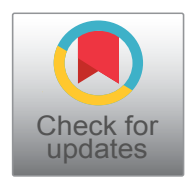

*Corresponding author: Lawrence M Asprec, MD, Resident Physician, Department of Rehabilitation Medicine, Thomas Jefferson University Hospital, Sidney Kimmel Medical College, 25 S. Ninth Street, Philadelphia, PA, 19107, USA, Tel: 215955-6585; 908-642-4754, Fax: 215-955-2311

\begin{abstract}
Introduction: We present a case of a man with unilateral adductor pollicis (AdP) and first dorsal interosseous (FDIO) muscle weakness with sparing of the hypothenar muscle group.

Case report: A 57-year-old man developed progressive weakness over 3 months in his right hand without sensory abnormalities. Clinical and electrodiagnostic examination revealed abnormalities in the FDIO. Clinical examination supported an abnormal AdP. Hypothenar muscle groups were intact, suggestive of an ulnar nerve lesion in the palm, an uncommon area of entrapment. MRI confirmed a ganglion cyst between the third and fourth metacarpal bases, compressing the deep palmar branch of the ulnar nerve. After removal of the cyst and neurolysis of the ulnar motor branch, AdP and FDIO strength improved five months postoperatively.

Conclusion: This deficit was caused by compression of the deep palmar branch of the ulnar nerve by a ganglion cyst in the mid palm, an uncommon area. Suspected ulnar neuropathies caused by etiologies at the wrist or palm, a combination of clinical evaluation, nerve conduction studies, and anatomic studies may reveal uncommon sites of compression.
\end{abstract}

\section{Keywords}

Ulnar neuropathy, Palmar lesion, Electromyography

\section{Introduction}

Lesions that cause ulnar neuropathy at or distal to Guyon's canal in the wrist are uncommon. The most common entrapment sites are at the retroepicondylar groove, humero-ulnar arcade and in between the two heads of the flexor carpi ulnaris [1]. The ulnar nerve has five major branches distal to the elbow, including the dorsal ulnar cutaneous nerve and palmar ulnar cutaneous nerve proximal to the wrist [1]. The ulnar nerve enters the hand above the flexor retinaculum radial to the pisiform bone and continues superficial to the piso-hamate ligament and occupies a groove on the medial aspect of the hook of the hamate [2]. It bifurcates into the superficial cutaneous and deep motor branch. The deep motor branch supplies the innervation to the hypothenar muscles and continues through the palm to innervate the interossei, the medial two lumbricals, the adductor pollicis (AdP) and the first dorsal interosseous (FDIO) muscles [2]. We present a case of a man with unilateral $\mathrm{AdP}$ and FDIO muscle weakness with sparing of the hypothenar muscle group. This deficit was caused by compression of the deep palmar branch of the ulnar nerve by a ganglion cyst in the mid palm. 


\section{Case Report}

A 57-year-old man presents with three months of progressive weakness of his dominant right hand without sensory abnormalities. He noted difficulties with writing, specifically painless inhibition of fine movements. Furthermore, he denied cervical pain or numbness, or weakness in his left hand. Physical examination revealed atrophy and decreased (1/5) strength the AdP and FDIO, with intact strength and bulk of the hypothenar muscle groups. There was a positive Froment's sign. Sensory examination was unremarkable on the dorsal and volar aspect of the hand and fingers.

Electrodiagnostic examination revealed normal peak latency and amplitude with stimulation of the ulnar sensory nerve. Distal latency and amplitude is normal with ulnar nerve stimulation and the active electrode over the abductor digiti minimi (ADM). However, stimulation of the ulnar nerve with the active electrode over the ipsilateral FDIO revealed decreased amplitude of the evoked response compared to normal as well as to the contralateral side. Ulnar nerve conduction velocity across the elbow was within normal limits. Electromyography of the FDIO also revealed diminished recruitment and large fibrillations while the abductor pollicis brevis, abductor digiti minimi, flexor carpi ulnaris, pronator teres, triceps and biceps was normal. The diagnosis was an ulnar nerve lesion in the mid palm which affected motor function only (Table 1 ).

MRI of the right hand confirmed a ganglion cyst between the third and fourth metacarpal bases, compressing the deep palmar branch of the ulnar nerve. Ulnar neurolysis and removal of the ganglion cyst was performed, and he subsequently completed a course of hand therapy. At the five month postoperative follow-up, motor strength of the AdP and FDIO improved to $+/ 5$. The Froment's sign was negative.

\section{Discussion}

Ulnar neuropathies of the wrist and hand are more uncommon than those at the elbow [1]. The most common etiology of distal ulnar nerve compression includes ganglion cysts derived from the pisiform-hamate-tri- quetral joint complex [3]. Other less common causes include trauma/occupational neuritis, tumors, lipomas, fibrous bands, and ulnar artery disease (arteritis, thromboangiitis) $[3,4]$ Less commonly seen are injuries secondary to leprosy, anomalous musculature, diabetes mellitus, vibrating tools, and bicycle handlebar palsy $[5,6]$. The differential diagnosis of ulnar neuropathy may include C8-T1 radiculopathy, lower trunk or medial cord plexopathy, peripheral neuropathy, and the more common ulnar neuropathy at the elbow [7]. Concurrent conditions, such as carpal tunnel syndrome may also confuse the diagnosis.

The clinical manifestation of this condition can present as a motor, sensory, or a combined nerve dysfunction. Shea and McClain classified three types of ulnar-nerve compression syndromes [3]. Type I involves a lesion proximal to or in Guyon's canal, and affects the superficial and deep nerve branches leading to both volar sensory deficit of the ulnar one and one half fingers and motor deficits in the ulnar intrinsic musculature. Type II involves a lesion in Guyon's canal at the hook of the hamate and near the origin of the abductor, flexor and opponens quinti muscles, and affects solely the deep branch, resulting in motor deficit without any sensory abnormality. Type III involves Guyon's canal near the hook of the hamate or in the palmaris brevis, affecting the superficial branch with resulting sensory deficit in the volar one and one half ulnar fingers without a motor deficit [3]. In this classification, the pattern of muscle group involvement in Type II lesions is not specified by the authors.

In order to address this deficit, $\mathrm{Wu}$, et al. expanded the syndromes into five types to distinguish syndromes that occur with compression of the deep motor branch proximal and distal to the innervation of the hypothenar musculature [8]. The first two types (Wu 1 and 2) describe mixed motor and sensor and purely sensory syndromes, corresponding to Shea and McClain's Type I and III respectively. In addition, a further definition of motor only deficit patterns (Shea and McClain's Type II) is provided by the following types: Wu 3 affects the hypothenar muscles and all of the ulnar intrinsic muscles, Wu 4 spares the hypothenar muscles, and Wu 5

Table 1: Motor and sensory nerve conduction studies of bilateral ulnar nerves. Note the decreased amplitude of the evoked response with stimulation of the ulnar nerve with active electrode over the FDIO compared to the ADM on the right side. Left-sided motor ulnar nerve conduction studies were within normal limits. Velocities were within normal limits and excluded from this data table.

\begin{tabular}{|c|c|c|}
\hline Stimulation Site & Distal Latency (ms) & Amplitude (mV) \\
\hline Ulnar Motor & Left/Right & \\
\hline Wrist to ADM & $3.3 / 3.3$ & 9.2 \\
\hline Below Elbow & $6.6 / 6.9$ & 8.4 \\
\hline Above Elbow & 8.3/8.8 & 9.0 \\
\hline Wrist to FDI & $3.4 / 3.0$ & 10.8/3.2 \\
\hline Ulnar Sensory & Left/Right & \\
\hline Wrist to $5^{\text {th }}$ Digit & $4.1 / 3.8$ & $24.5 / 17.2$ \\
\hline
\end{tabular}


spares the hypothenars, lumbricals and interossei, and only affects the AdP and FDIO [8]. Our patient's ulnar neuropathy generally falls under Shea \& McClain's Type II class, but is better and more clearly defined as a Wu 5 injury [8].

While the diagnosis of a motor only ulnar nerve palsy at the wrist is not uncommon, our case is unique in that the ulnar palsy occurred distal to the takeoff of the hypothenar and lumbrical/interossei branches secondary to a compressive ganglion cyst, affecting only the AdP and FDIO. This neuropathy would be classified as a Wu 5 type. In Wu's review of 55 literature cases, only one could be classified as a Wu 5. Capitani and Beer describe three cases of these subtypes, but could not distinguish between a $\mathrm{Wu} 4$ or 5 types, and all were secondary to athletic injuries [6]. Chiodo describes one and Seror seven similar cases, but like those described by Capitani, the neuropathy could not be distinguished between Wu 4 or 5 types, and the injuries occurred due to an athletic injury $[7,9]$. Only one other report describes a distal ulnar motor compression secondary to a compressive lesion [5]. Nevertheless, the neuropathy could not be distinguished between a Wu 4 or 5 type, but given the location of the cyst on the ulnar aspect of the wrist, this was likely to be a Wu 4 injury. Finally, no results of treatment are provided in that report.

Stimulation of the ulnar nerve with the active electrode over the FDIO revealed a decreased motor amplitude; all other nerve conduction studies were within normal limits on the affected side. Aysal, et al. also suggested including motor recording from FDIO at stimulation points proximal to Guyon canal, as well as distal to the canal with mid-palmar stimulation. This is technically difficult with a mid-palmar lesion since stimulation distal to the injury may not be feasible secondary to the proximity to the first dorsal interosseous [10]. Because we did not perform a mid-palmar stimulation to the FDIO, we could not state whether or not there was a conduction block [11]. A poor prognosis could not be made on the basis of a low amplitude potential obtained with stimulation proximal to the wrist, without ruling out a conduction block. If the amplitude normalizes with stimulation distal to the wrist or if there is a normal amplitude with stimulation proximal to the wrist, prognosis is predicted to be good. If the amplitude of the evoked response were to be low with stimulation in the midpalm, the prognosis would be poor. The degree of axonal compromise can't be quantified based on the needle study $[11,12]$. Thus, even though we found denervation on the needle study in our patient, we could not estimate the degree of axonal compromise and could not make a prognostication. Given the fact that the patient made a rapid recovery after surgery would strongly suggest a significant demyelinating more than axonal component to this lesion.

\section{Conclusion}

In summary, our case study demonstrates the need for NCS and EMG of the FDI when exploring etiologies of ulnar neuropathies thought to be at the wrist and palm, based on the clinical presentation. The ability to prognosticate based on the NCS may be limited if the amplitude is low unless a mid-palmar stimulation can establish whether the lesion is secondary to axonal or myelin injury. This is not always technically feasible. This is critical since a misdiagnosis or incorrect prognosis may change the treatment. Anatomic studies should be ordered to identify a compressive lesion and complement the electrodiagnostic evaluation.

\section{Clinical Message}

When exploring suspected ulnar neuropathies caused by etiologies at the wrist or palm, a combination of clinical evaluation, nerve conduction studies, and anatomic studies may reveal uncommon sites of compression.

\section{Statement of Informed Consent}

The patient was informed that data concerning the case would be submitted for publication and they agreed and consented.

\section{Acknowledgments}

The authors declare that they have no competing interests or any sources of financial support received.

\section{References}

1. Olney RK, Hanson M (1988) Ulnar neuropathy at or distal to the wrist. Muscle Nerve 11: 828-832.

2. Ebeling P, Gillitt RW, Thomas PK (1960) A clinical and electrical study of ulnar nerve lesion in the hand. J Neurol Neurosurg Psychiatry 23: 1-9.

3. Shea JD, McClain EJ (1969) Ulnar-Nerve Compression Syndromes at and below the wrist. J Bone Joint surg Am 51: 1095-1103.

4. Duggal A, Anastakis DJ, Salonen D, Becker E (2006) Compression of the deep palmar branch of the ulnar nerve by a ganglion Hand 1: 98-101.

5. Jacob A, Moorthy TK, Thomas SV, Sarada C (2005) Compression of the deep motor branch of the ulnar nerve : An unusual cause of pure motor neuropathy and hand wasting. Arch Neurol 62: 826-827.

6. Capitani D, Beer S (2002) Handlebar palsy - A compression syndrome of the deep terminal (motor) branch of the ulnar nerve in biking. J Neurol 249: 1441-1445.

7. Chiodo A, Chadd E (2007) Ulnar neuropathy at or distal to the wrist: Traumatic versus cumulative stress cases. Arch Phys Med Rehabil 88: 504-512.

8. Wu JS, Morris JD, Hogan GR (1985) Ulnar neuropathy at the wrist: Case report and review of literature. Arch Phys Med Rehabil 66: 785-788. 
9. Seror $P$ (2015) Ulnar nerve lesion at the wrist and sport: $A$ report of 8 cases compared with 45 non-sport cases. Ann Phys Rehabil Med 58: 104-109.

10. Aysal F, Mutluay B, Soysal A, Koksal A, Baybas S, et al. (2014) Ulnar nerve compression at the wrist : Diagnostic role of palmar stimulation. J Clin Neurophysiol 31: 444-449.
11. Robinson LR (2000) Traumatic injury to peripheral nerves. Muscle Nerve 23: 863-873.

12. Friederich JM, Robinson LR (2011) Prognostic indicators from electrodiagnostic studies for ulnar neuropathy at the elbow. Muscle Nerve 43: 596-600. 